\title{
Einleitung: Alltagspraktiken des Publikums
}

\author{
Stephan Habscheid • Christine Hrncal • Raphaela Knipp • Erika Linz
}

Online publiziert: 17. Januar 2017

(C) J.B. Metzler, Part of Springer Nature, Springer-Verlag GmbH 2016

Ästhetische Artefakte und Ereignisse, sei es in der Kunst oder der populären Kultur, sind ohne Publikum nicht denkbar. Wie aber das Publikum für die Gegenwart begrifflich zu bestimmen ist, wie es sich im Umgang mit Kunstwerken und -vollzügen wahrnehmend und rezipierend verhält, vor allem: wie es die Künste und sich selbst während oder nach der Rezeption - eingebettet in räumlich, zeitlich, dinglich, physisch situierte Alltagspraktiken ${ }^{1}$ - kommunikativ formiert und welche Relevanz vor diesem Hintergrund »dem Publikum« für die Kunst und in der Gesellschaft zukommt, muss nach wie vor als weitgehend ungeklärt gelten.

In begriffsgeschichtlicher Perspektive handelt es sich beim Publikum nach gängiger Auffassung zunächst um eine »Kategorie der bürgerlichen Gesellschaft« (Ha-

\footnotetext{
1 Praktiken - als >Lebensformen $<$ und $>$ Sprachspiele $<$ (Wittgenstein) - verbinden nach Theodore R. Schatzki (1996, S. 12 f.) praktisches Tun, damit einhergehende materielle Arrangements, die situierte Verwendung von sprachlichen und anderen Zeichen in der Interaktion und die >innere< Ordnung im Geist der Beteiligten; sie bilden gesellschaftstheoretisch den Schnittpunkt zwischen Handeln und - beweglichen Institutionen und Strukturen (ebd., S. 11 f.), und sie greifen als »geschicktes Bewältigen« (Sharrock 2012, S. 60) auf verschiedene Arten von Wissen und Können zurück; vgl. dazu näher Habscheid (2016).
}

\author{
S. Habscheid $(\bowtie) \cdot$ C. Hrncal \\ Universität Siegen, 57068 Siegen, Deutschland \\ E-Mail: habscheid@germanistik.uni-siegen.de \\ C. Hrncal \\ E-Mail: hrncal@germanistik.uni-siegen.de \\ R. Knipp \\ Ruhr-Universität Bochum, 44810 Bochum, Deutschland \\ E-Mail: raphaela.knipp@rub.de \\ E. Linz \\ Rheinische Friedrich-Wilhelms Universität Bonn, 53113 Bonn, Deutschland \\ E-Mail: elinz@uni-bonn.de
}


bermas 1990), die im Kern üblicherweise wie folgt charakterisiert wird (Kammerer 2012): Seit dem späteren 18. Jahrhundert überschneiden sich im Begriff des Publikums ästhetische mit gesellschaftlich-politischen Diskursen. Die Verschränkung von politischem und literarischem Diskurs bildete eine Grundlage dafür, dass sich die Öffentlichkeit »vom Adressaten obrigkeitsstaatlicher Rechtsakte zur gebildeten bürgerlichen Gesellschaft« wandeln konnte (Hölscher 1978/2004, S. 434, zitiert nach Kammerer 2012a, S. 7). In der späteren Entwicklung scheint dieses Konzept des Publikums nicht mehr zu greifen, so dass nun typischerweise von einer »Krise « der bürgerlichen Öffentlichkeit die Rede ist (Kammerer 2012a, S. 8).

Wortgeschichtlich entspricht dieser Veränderung, dass mit dem Ausdruck >Publikum< heute oft lediglich »lokale Ansammlungen von Zuschauern und Zuschauerinnen, Konsumenten und Konsumentinnen « bezeichnet werden, die an einem KulturEvent teilnehmen beziehungsweise von den Veranstaltern dieses Events als »Zielgruppen« adressiert werden (Kernbauer 2012, S. 63). Der heutige Begriff des Publikums habe daher nicht mehr »denjenigen Nimbus gemeinschaftlicher politischer und ästhetischer Urteilskraft, die einmal die Öffentlichkeit in den für die Kunsttheorie besonders einflussreichen Darstellungen [...] auszeichnete« (ebd.).

$\mathrm{Zu}$ dieser Entwicklung trug auch der kunsttheoretische Diskurs das seine bei: Hier wurde das Publikum begrifflich häufig zu einem passiven, kollektiven >Patiens $<$ abgewertet und dem individuellen, schaffenden Künstler-Genie als >Agens $<$ gegenüber gestellt (vgl. Kammerer 2012a, S. 8). Zumal unter den Bedingungen der Massenkultur die Relevanz eines Kunstwerks programmatisch als >autonom< erachtet wurde gegenüber der Resonanz, die es beim breiten Publikum findet: »Kein Kunstwerk « könne »in Kategorien der Kommunikation« beschrieben und erklärt werden (Adorno 1970, S. 167). Dagegen wird in der jüngeren Kunsttheorie - wie auch bereits in früheren emanzipatorischen Ansätzen - (wieder) verstärkt danach gefragt, wie das Publikum sozial und politisch zu aktivieren wäre, Bettina Brandl-Risi spricht gar von der Idee einer »großen Mobilmachung des Publikums [...], die sowohl auf dem Theater wie auch in der neu entstehenden Theaterwissenschaft als Entdeckung des Zuschauers gefeiert wurde « (Brandl-Risi 2012, S. 74). Vor diesem Hintergrund setzt etwa die so genannte >Relationale Ästhetik < auf die Partizipation des Publikums bei der künstlerischen Herstellung sozialer Beziehungen; allerdings bleibt hier, wie Eva Kernbauer (2012, S. 64) einwendet, oft unscharf, worauf die Annahme der politischen Wirksamkeit dieser Praxis genau beruhe. - Insgesamt, so kann man mit Dietmar Kammerer resümieren, »bleibt das Publikum eine hartnäckig eigensinnige Größe, der weder mit empirischen Merkmalserhebungen noch mit impliziten Lektüren und semantisch-symbolischen Konstruktionen beizukommen ist. [...] Logiken der Marktforschung und des >Audience Development< tun ihr Übriges, um den Zuschauer auf seine Gewohnheiten, seine Bedürfnisse und seine (monetären) Ressourcen zu reduzieren [...]«(Kammerer 2012a, S. 8).

Vor diesem Hintergrund wird von kulturwissenschaftlicher Seite vorgeschlagen, das Konzept >Publikum «metaphorisch zu entwerfen als »Beschreibung für einen Durchgangs- oder Umschlageplatz [...], der selbst unbestimmt zu halten wäre: als denjenigen Punkt, an dem Kunst und Öffentlichkeit konvergieren und Eigenschaften, Inhalte, Wahrnehmungen, usw. austauschen, vermitteln, transferieren oder anbieten « (ebd., S. 8); andere Metaphern beschreiben das Publikum als ein »Loch [...]: als et- 
was, das sich widersetzt, gefüllt zu werden« (ebd., S. 9, unter Bezug auf Raunig 2005) oder als einen »Raum der Möglichkeit jenseits der Begrenzungen von Institutionen oder Einzelkünsten« (Kammerer 2012a, S. 9). Gemeinsam ist all diesen Bildern, dass mit ihnen versucht wird, tradierte Asymmetrien aufzulösen (Kernbauer 2012, S. 67 f.): zwischen denen, die sprechen und denen, die hören; denen, die aktiv etwas geben, und denen, die passiv etwas empfangen; denen, die in ästhetischer Form belehren, und denen, die belehrt werden. Dass selbst in >massenmedialen< Kommunikationsprozessen dem Publikum ein aktiver und produktiver Part mit durchaus kritischem Potenzial zukommt, bildet eine grundlegende Annahme der kulturwissenschaftlichen Audience Studies (klassisch Morley 1980). ${ }^{2}$

Die Frage, ob, inwieweit und wie genau sich derartige Konzeptualisierungen des Publikums in Form alltäglicher, kommunikativer Praktiken im Umgang mit Kunst empirisch konkretisieren, steht im Mittelpunkt dieses Heftes. ${ }^{3}$ Der Fokus der Beiträge aus der Linguistik, der Soziologie sowie den Literatur- und Kulturwissenschaften liegt auf den in sozialer Interaktion verankerten Alltagspraktiken des Publikums im Kontext der >Kunstkommunikation< in Theater (vgl. Linz/Hrncal/Schlinkmann in diesem Heft), städtischem Raum (Knipp), auf Festivals und Großveranstaltungen (Knoblauch), im Fernsehstudio (Holly) - sowie nicht zuletzt in Verbindung mit dem Social Web (Müller/Stegmeier). Ziel des Heftes ist es, diese unterschiedlichen Ausprägungen von Alltagspraktiken durch empirische Untersuchungen 1. über die verschiedenen Institutionen und Felder hinweg zu vergleichen sowie 2. zu Theorieangeboten des literatur-, kultur- und sozialwissenschaftlichen Diskurses über das Verhältnis von Kunst und Gesellschaft erörternd in Beziehung zu setzen.

Unter Kunstkommunikation verstehen wir - anknüpfend an Filk/Simon (2010) und Hausendorf (2007, 2011) - nicht nur die Kommunikation mit Kunst und durch Kunst (Hausendorf 2007, S. 19; Müller/Kluwe 2012, S. 4, unter Bezug auf Luhmann 1995), sondern auch die Kommunikation über Kunst. Beide Formen der Kunstkommunikation sind in kunstsoziologischer Perspektive eng miteinander verwoben (siehe Hausendorf 2007, S. 19). Selbst Adornos Diktum, wonach »kein Kunstwerk in Kategorien der Kommunikation zu beschreiben und zu erklären« ist (Adorno 1970, S. 167), trägt in paradoxer Weise als Kommunikation über Kunst zum kommunikativen Umgang mit Kunst bei (vgl. Müller/Kluwe 2012, S. 2 f.).

$\mathrm{Zu}$ den Implikationen der systemsoziologischen Beobachtungen Niklas Luhmanns zählt aber auch die Ausdifferenzierung eines spezifischen Kunstpublikums als Teil eines autonomen Funktionssystems und Pendants zu ebenso spezifizierten Funktionsrollen (Autoren, Künstler, Dichter etc.): »Jetzt produziert die Kunst ihr eigenes Publikum, und die Frage kann nur noch sein, wer daran partizipieren

\footnotetext{
2 Die frühen Ansätze der Cultural Studies beziehungsweise der Audience Studies zählen mit zu den ersten, die die Rolle des Publikums nicht auf die der passiven Konsumentinnen und Konsumenten reduzieren. Ihre Arbeiten antworteten damals auf klassische Theorien der Öffentlichkeit und ihres Publikums mit empirischen Mikrostudien, die Publikums- und Rezeptionspraktiken an den Orten ihrer Entstehung, z.B. in häuslichen Kontexten, zu erfassen suchten.

3 Bei dem vorliegenden Heft handelt es sich um den Auftakt einer Doppelausgabe der Zeitschrift für Literaturwissenschaft und Linguistik zum Themenschwerpunkt >Publikumく. Der Fokus dieser Ausgabe liegt auf der Beschäftigung mit konkreten Publikumspraktiken. Das Folgeheft erscheint voraussichtlich im Dezember 2017 unter dem Titel »Konstruktionen des Publikums«.
} 
kann« (Luhmann 2008, S. 161). Zwar wird man heute, etwa im Social Web, auch im Falle abgelegenster Produktionen Spuren von kommunikativer Relevanz nachweisen können, doch belegt dies nur, dass die Antwort auf Luhmanns Frage heute wie auch schon im 18. Jahrhundert immer nur partiell gegeben werden kann: insofern niemand an »Kunst« schlechthin partizipiert, sondern stets nur bestimmte Werke rezipiert und das Publikum daher in zahlreiche Segmente zersplittert. Auch für postdramatisches Theater oder Publikumsbeschimpfungen gibt es Fankreise, die angemessene Rezeptionspraktiken hervorbringen, aber für jedes Kunstwerk bedeutet dies im Umkehrschluss, dass Anschlussfähigkeit und Resonanz unwahrscheinlich sind. Die Ausdifferenzierung bestimmter Publika für bestimmte Kunstofferten (etwa Freunde neuer Musik oder Abonnenten eines Theaters) kompensiert diese Unwahrscheinlichkeit nur zum Teil, denn letztlich muss in der Moderne davon ausgegangen werden, dass Kunst für anonyme Rezipienten produziert wird (vgl. Luhmann 2008, S. 326 f.). Ob ein Werk ein Publikum attrahieren kann und welche Praktiken des Publikums es womöglich stimuliert oder nach sich zieht, bleibt daher aus systemtheoretischer Sicht eine grundsätzlich offene Frage, der man auch mit Organisation nicht beikommt (vgl. Werber 2002).

Umso mehr stellt sich die Frage nach den grundlegenden medialen und interaktionalen Bedingungen, die Kommunikation im Raum der Kunst ermöglichen und elementar strukturieren. Wie empirisch leicht festzustellen ist, stoßen Kunst und populäre Kultur in vielfältiger Hinsicht Kommunikation an (vgl. Filk/Simon 2010, S. 23), ob im Kunstmuseum (vgl. Heath/vom Lehn 2004; Hausendorf 2007) oder vor dem heimischen Fernsehapparat (Holly/Püschel/Bergmann 2001). Im Einzelnen kann man mit Dirk Baecker (2013, S. 17) heuristisch davon ausgehen, dass die Spezifik der verschiedenen Künste in den jeweiligen Dispositiven begründet liegt, in denen sie Wahrnehmungsgewohnheiten des Publikums - und damit eingespielte kommunikative Ordnungsstrukturen - ästhetisch in Frage stellen und damit Anlässe für Kommunikation über Kommunikation schaffen. Darüber hinaus dient die kommunikative Auseinandersetzung mit Kunst, wie Müller und Kluwe (2012, S. 1, 4, $7 \mathrm{f}$.) hervorheben, der Konstruktion von >eigener< und >fremder< Identität, in Kollektiven und Gruppen sowie als Teil individueller Bildungs- und Sozialisationsprozesse.

Die Beiträge von Raphaela Knipp und Niels Werber adressieren die Frage nach Publikumspraktiken aus literatur- und kulturwissenschaftlicher Perspektive. Sie machen deutlich, dass die Beschäftigung mit Alltagspraktiken des rezeptiven Umgangs mit literarischen Texten nach wie vor ein Desiderat innerhalb der Literaturwissenschaften darstellt. Niels Werber nähert sich der Rolle des Publikums dabei über Forschungen zum Populären. Unter Rekurs auf soziologische Ansätze von Niklas Luhmann und Urs Stäheli plädiert sein Beitrag für eine Verschränkung von $\mathrm{Pu}-$ blikums- und Populärkulturforschung, die unter anderem die Rolle von Affekten beziehungsweise affektiven Bindungen stärker berücksichtigt. Raphaela Knipp zeigt anhand ihrer Forschungen zum Literaturtourismus auf, wie sich mit einem qualitativ-ethnografischen Ansatz konkrete Publikumspraktiken empirisch untersuchen lassen und wie ein solcher Ansatz den Blick auf die Rezeption literarischer Werke verändern und erweitern kann.

Marcus Müller und Jörn Stegmeier beschäftigen sich in ihrem Beitrag mit alltäglichen Praktiken der Kunstrezeption in sozialen Medien, konkret auf Twitter. In 
qualitativen Analysen von Tweets gehen die Autoren der Frage nach, ob und wie sich Nutzerinnen und Nutzer in diesem Medium aktiv mit Kunst auseinandersetzen und sozial positionieren. Gegenstand des gesprächslinguistisch ausgerichteten Beitrags von Erika Linz, Christine Hrncal und Eva Schlinkmann sind die Aneignungspraktiken des Publikums im Rahmen von Theaterbesuchen. Der Beitrag zeigt, wie im Theater die Anschlusskommunikation im Foyergespräch dazu genutzt wird, angeregt durch die Bühnenerlebnisse miteinander ins Gespräch zu kommen, individuelle, ästhetische Erfahrungen interaktiv abzugleichen und auszuhandeln sowie auf die gesellschaftliche Wirklichkeit zu übertragen.

Einem weitgehend vernachlässigten Gegenstand der Publikumsforschung widmet sich auch der soziologisch orientierte Beitrag von Hubert Knoblauch, den Publikumsemotionen. Anhand von videografischen Untersuchungen von Präsenzpublika im Kontext von Sportereignissen und religiösen Großveranstaltungen zeigt Knoblauch, wie sich Publikumsemotionen als Formen fassen lassen, die durch die kollektive Koordination kommunikativer Handlungen hervorgebracht werden. Auch der Beitrag von Werner Holly nähert sich dem Begriff des Publikums über das Feld der Populärkultur. Ausgehend von seinen linguistischen Arbeiten zur Fernsehrezeption befasst sich Holly am Beispiel von Ausschnitten aus Polit-Talkshows mit Praktiken der akustischen und optischen Wahrnehmbarmachung des Studiopublikums, die darauf zielen, Partizipation zu suggerieren und eine Brücke zu den Zuschauern »draußen an den Bildschirmen« zu inszenieren.

Die Beiträge dieser Ausgabe resultieren aus der interdisziplinären Tagung »Alltagspraktiken des Publikums: Theater, Literatur, Kunst, Populärkultur«, die am 29. Februar und 01. März 2016 im Apollo-Theater Siegen stattfand. Die Tagung geht auf eine Kooperation des DFG-Projektes »Theater im Gespräch. Sprachliche Kunstaneignungspraktiken in der Theaterpause « unter der Leitung von Stephan Habscheid (Universität Siegen) und Erika Linz (Universität Bonn), des Siegener DFG-Graduiertenkollegs »Locating Media« sowie des Handbuch-Projektes »Sprache in der Kunstkommunikation« unter der Leitung von Marcus Müller (TU Darmstadt) und Heiko Hausendorf (Universität Zürich) zurück. Unser Dank gilt allen Mitarbeiterinnen und Mitarbeitern der Projekte für ihr inhaltliches und administratives Engagement sowie natürlich allen Referentinnen und Referenten der Tagung.

\section{Literatur}

Adorno, Theodor W.: Ästhetische Theorie. Frankfurt a.M. 1970.

Baecker, Dirk: Wozu Theater? Berlin 2013.

Brandl-Risi, Bettina: »Genuss und Kritik. Partizipieren im Theaterpublikum«. In: Dietmar Kammerer (Hg.): Vom Publicum. Das Öffentliche in der Kunst. Bielefeld 2012, S. 73-90.

Filk, Christian/Simon, Holger: »〉Wie ist Kunst möglich?< Zur Konstitution von Kunstkommunikation«. In: Dies. (Hg.): Kunstkommunikation: »Wie ist Kunst möglich? «Beiträge zu einer systemischen Medienund Kunstwissenschaft. Berlin 2010, S. 17-35.

Habermas, Jürgen: Strukturwandel der Öffentlichkeit. Untersuchungen zu einer Kategorie der bürgerlichen Gesellschaft. Mit einem Vorwort zur Neuauflage 1990. Frankfurt a.M. 1990.

Habscheid, Stephan: »Handeln in Praxis. Hinter- und Untergründe situierter sprachlicher Bedeutungskonstitution«. In: Arnulf Deppermann/Helmuth Feilke/Angelika Linke (Hg.): Sprachliche und kommunikative Praktiken. (= Jahrbuch des Instituts für deutsche Sprache 2015). Berlin/New York 2016, S.127151 . 
Hausendorf, Heiko: »Die Sprache der Kunstkommunikation und ihre interdisziplinäre Relevanz«. In: Ders. (Hg.): Vor dem Kunstwerk. Interdisziplinäre Aspekte des Sprechens und Schreibens über Kunst. München 2007, S. 17-51.

Hausendorf, Heiko: »Kunstkommunikation«. In: Stephan Habscheid (Hg.): Handlungsmuster, Textsorten, Oberflächen. Linguistische Typologien der Kommunikation. Berlin/New York 2011, S. 509-535.

Heath, Christian/vom Lehn, Dirk: »Configuring Reception. (Dis-)Regarding the >Spectator< in Museums and Galleries«. In: Theory, Culture \& Society 21/6 (2004), S. 43-65.

Holly, Werner/Püschel, Ulrich/Bergmann, Jörg (Hg.): Der sprechende Zuschauer. Wie wir uns Fernsehen kommunikativ aneignen. Wiesbaden 2001.

Hölscher, Lucian: »Öffentlichkeit«. In: Otto Brunner u.a. (Hg.): Geschichtliche Grundbegriffe. Historisches Lexikon zur politisch-sozialen Sprache in Deutschland. Bd. 4. Stuttgart [1978] 2004, S. 413467.

Kammerer, Dietmar (Hg.): Vom Publicum. Das Öffentliche in der Kunst. Bielefeld 2012.

Kammerer, Dietmar: »Vorwort. Vom Publicum«. In: Ders. (Hg.): Vom Publicum. Das Öffentliche in der Kunst. Bielefeld 2012a, S. 7-11.

Kernbauer, Eva: »Das Publikum in der kunsttheoretischen Tradition: Wege zur Öffentlichkeit (und zurück)«. In: Dietmar Kammerer (Hg.): Vom Publicum. Das Öffentliche in der Kunst. Bielefeld 2012, S. 49-71.

Luhmann, Niklas: Die Kunst der Gesellschaft. Frankfurt a.M. 1995.

Luhmann, Niklas: Schriften zur Kunst und Literatur. Hg. von Niels Werber. Frankfurt a.M. 2008.

Morley, David: The >Nationwide<Audience: Structure and Decoding. London 1980.

Müller, Marcus/Kluwe, Sandra: »Kunstkommunikation und Identität«. In: Dies. (Hg.): Identitätsentwürfe in der Kunstkommunikation. Studien zur Praxis der sprachlichen und multimodalen Positionierung im Interaktionsraum Kunst. Berlin/New York 2012, S. 1-22.

Raunig, Gerald: »Jenseits der Öffentlichkeit«. In: Ders./Ulf Wuggening (Hg.): Publicum. Theorien der Öffentlichkeit. Wien 2005, S. 225-232.

Schatzki, Theodore R.: Social Practices. A Wittgensteinian Approach to Human Activity and the Social. Cambridge 1996.

Sharrock, Wes: »Regelfolgen: Alles oder nichts?« In: Ruth Ayaß/Christian Meyer (Hg.): Sozialität in Slow Motion. Theoretische und empirische Perspektiven. Festschrift für Jörg Bergmann. Wiesbaden 2012, S. 59-70.

Werber, Niels: »Kommunikation ohne Interaktion. Thesen zu einem zweiten >Strukturwandel< der Massenmedien«. In: Jürgen Fohrmann/Arno Orzessek (Hg.): Zerstreute Öffentlichkeiten. München 2002, S. 43-52. 